\title{
Some anomalies of mesosphere/lower thermosphere parameters during the recent solar minimum
}

\author{
Ch. Jacobi ${ }^{1}$, P. Hoffmann ${ }^{1}$, M. Placke ${ }^{2}$, and G. Stober ${ }^{2}$ \\ ${ }^{1}$ Institute for Meteorology, University of Leipzig, Stephanstr. 3, 04103 Leipzig, Germany \\ ${ }^{2}$ Leibniz Institute of Atmospheric Physics at the Rostock University, Schlossstraße 6, 18225 Kühlungsborn, Germany
}

\begin{abstract}
The recent solar minimum has been characterized by an anomalous strong decrease of thermospheric density since 2005. Here we analyze anomalies of mesosphere/lower thermosphere parameters possibly connected with this effect. In particular, nighttime mean LF reflection heights measured at Collm, Germany, show a very strong decrease after 2005, indicating a density decrease. This decrease is also visible in mean meteor heights measured with VHF meteor radar at Collm. This density decrease is accompanied by an increase of gravity wave (GW) amplitudes in the upper mesosphere and a decrease in the lower thermosphere. On the decadal scale, GWs are negatively correlated with the background zonal wind, but this correlation is modulated in the course of the solar cycle, indicating the combined effect of GW filtering and density decrease.
\end{abstract}

\section{Introduction}

It is widely known that solar variability influences the atmosphere (Gray et al., 2010), e.g., the dynamics of the middle and upper atmosphere. In particular, search for an effect of the 11-year solar Schwabe cycle has been undertaken, for example, to explain part of the observed variability of mesosphere and lower thermosphere (MLT), which can be studied by radars. Indeed, indication for a solar effect has been found in MLT radar wind time series over Central Europe (Jacobi and Kürschner, 2006; Keuer et al., 2007). This effect is more pronounced in summer than in winter owing to the more disturbed middle atmosphere during winter, and essentially consists in a stronger mesospheric jet during solar maximum. In the summer lower thermosphere, above the mean wind reversal, this results in weaker westerly winds during solar maximum, i.e. a negative correlation between mean wind and solar flux. This effect is mainly caused by the reaction of the stratosphere and mesosphere on solar flux

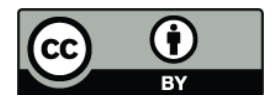

Correspondence to: Ch. Jacobi (jacobi@uni-leipzig.de) changes, and consequently decreases with altitude (Keuer et al., 2007).

The lower thermosphere wind reversal is owing to momentum deposition of gravity waves (GWs) in the upper mesosphere. According to linear theory, GWs with wind speed amplitudes exceeding the intrinsic phase speed, i.e., the difference between phase speed and mean wind speed, break and transfer part of their momentum to the background flow. This means that a GW amplitude remains at the breaking level, equaling the intrinsic phase speed, which is called a saturated GW. During summer/winter, strong mesospheric easterlies/westerlies prevent westward/eastward travelling GWs from propagating to the upper mesosphere, because their intrinsic phase speeds become small or even zero (a so called critical line). This means that in summer/winter only eastward/westward travelling GWs remain in the MLT region. Since these waves are still saturated, during sum$\mathrm{mer} /$ winter a stronger mesospheric easterly/westerly jet is expected to be connected with larger GW amplitudes. Consequently, in the upper mesosphere a positive correlation between solar flux and GW amplitudes is expected. Jacobi et al. (2006) have reported such a connection using GW proxy from Collm E-region drift measurements.

Solar cycles can differ from one to another. Especially, the recent solar minimum has been extremely extended and extraordinarily deep. Consequently, it led to extreme upper atmosphere reactions, in particular a decrease of thermospheric density (Emmert et al., 2010; Solomon et al., 2010) which exceeds the expectations that would have been based on conventional solar indices like the sunspot number or F10.7.

Lower ionospheric electron density reacts on the solar cycle, which leads to an 11-year modulation of radio wave reflection heights (e.g., Bremer and Berger, 2002; Bremer, 2005). These have been observed, e.g., by Kürschner and Jacobi (2003) who found that the Collm LF reflection heights $(177 \mathrm{kHz}$, distance to transmitter about $160 \mathrm{~km})$ are about $2 \mathrm{~km}$ lower during solar maximum than during solar minimum. This result has confirmed earlier findings (Entzian, 1967). Owing to the increased ionisation during solar maximum, however, this effect is superposed by thermal shrinking 
of the mesosphere during solar minimum, since the middle atmosphere has a solar cycle signal of about $2 \mathrm{~K}$ difference between maximum and minimum (Keckhut et al., 1995). This thermal shrinking is usually overcompensated by increased ionisation. We are interested, whether the recent extreme solar minimum has led to anomalous signatures either in MLT wind or density. We focus on the summer MLT, which is not that much influenced by stratospheric planetary wave activity.

\section{Measurements}

\subsection{Collm LF lower ionospheric drifts, reflection heights, and GW estimates}

At Collm Observatory, MLT winds have been obtained by D1 LF radio wind measurements from 1959-2008, using the ionospherically reflected sky wave of three commercial radio transmitters. The data are combined to half-hourly zonal and meridional mean wind values. The virtual reflection heights have been estimated since late 1982 using measured travel time differences between the separately received ionospherically reflected sky wave and the ground wave (Kürschner et al., 1987). More details of the Collm LF system are given in Jacobi (2011).

Since the LF reference height changes in the course of the day, a continuous time series at some fixed height is not available and consequently GW spectra cannot be calculated. However, using the method presented by Gavrilov et al. (2001a, b), horizontal wind fluctuations can be obtained which may serve as GW proxy. In brief, the method uses differences between two consecutive half-hourly means of the horizontal wind, if the mean reflection height does not change more than $1 \mathrm{~km}$ between these time intervals. Calculation of such differences combined with previous halfhourly averaging of the data is equivalent to a numerical filter passing harmonics with periods of $0.7-3 \mathrm{~h}$ with a maximum at about $1 \mathrm{~h}$ (Gavrilov et al., 2001a). Then the squared differences are averaged for $10 \mathrm{~km}$ height ranges during the time interval under consideration. Jacobi et al. (2006) has used this method to analyze the Collm dataset from 1984-2003. They found an 11-year solar cycle with larger GW amplitudes during solar maximum, but their dataset did not include the recent solar minimum.

\subsection{Collm meteor radar}

At Collm Observatory $\left(51.3^{\circ} \mathrm{N}, 13^{\circ} \mathrm{E}\right)$, a SKiYMET meteor radar (MR) is operated on $36.2 \mathrm{MHz}$ to measure horizontal winds in the $80-100 \mathrm{~km}$ height range, meteor rates and heights, and further meteor parameters since August 2004. The radar and the hourly wind detection are described in Jacobi (2011). Monthly mean wind parameters are obtained from one month of half-hourly winds applying a multiple regression analysis including the mean wind and tidal compo- nents. Based upon 2-hourly means, GW variances and fluxes are obtained according to Hocking (2005) by projecting the 2-hourly mean GW fluxes in a $3 \mathrm{~km}$ height gate to the radial direction of the respective meteor, and minimizing the difference between projected and measured meteor trail drift variances. Details can be found in Placke et al. (2011). The data are averaged to obtain 3-monthly means for each height gate under consideration.

\subsection{GW potential energy from SABER}

The SABER instrument on the TIMED satellites (Russell et al., 1999; Mertens et al., 2001, 2004) scans the atmosphere from about $52^{\circ}$ of one hemisphere to $83^{\circ}$ of the other. This latitude range is reversed by a yaw manoeuvre every 60 days. Due to the sun-synchronous orbital geometry the spacecraft passes the equator always at the same local time (12:00 LT) on the day side. Each single temperature profile, having a vertical resolution of $0.5 \mathrm{~km}$, is high-pass filtered to analyze waves with vertical wavelength of up to $6 \mathrm{~km}$. These filtered data represent deviations $T^{\prime}$ of temperature from the background. Their average specific potential energy in a $10 \mathrm{~km}$ vertical window is obtained through

$E_{\mathrm{p}}=\frac{1}{2}\left(\frac{g}{N}\right)^{2} \overline{\left(\frac{T^{\prime}}{\bar{T}}\right)^{2}}$,

with the bar denoting averaging over the respective $10 \mathrm{~km}$ window, and $N$ and $g$ being the buoyancy frequency and the acceleration due to gravity, respectively. The vertical profile of GW activity is obtained through shifting the data window by $1 \mathrm{~km}$ step and repeating the procedure. We use seasonal means at $45^{\circ} \mathrm{N}$ of zonally averaged potential energy here. The method of potential energy determination has frequently been applied to GPS radio occultations (e.g., Fröhlich et al., 2007). Note that limb scanning of the atmosphere by SABER only reveals certain parts of the GW spectrum due to the integration along the line of sight (Preusse et al., 2006). Another limitation is made by the chosen vertical filter, which allows only GWs of short wavelengths to be studied.

\section{GW proxy decadal variability}

Jacobi et al. (2006) had shown that there is a solar cycle influence on GW activity as measured by LF over Collm. Figure 1 presents the summer (JJA) mean time series of GW variance $\zeta^{\prime 2}=u^{\prime 2}+v^{\prime 2}$ at $100 \mathrm{~km}$ virtual height, which represents approximately $91 \mathrm{~km}$ real height (Jacobi, 2011). The data are an update from Jacobi et al. (2006). The 13-monthly mean sunspot number is added. Clearly, there is a solar cycle in the GW variance, and from visual inspection a decreasing longterm trend is also visible. We thus added, as a red solid line, a least squares fit of a linear trend superposed by a solar cycle

$\zeta^{\prime 2}=a+b \times y r+c \times R$, 


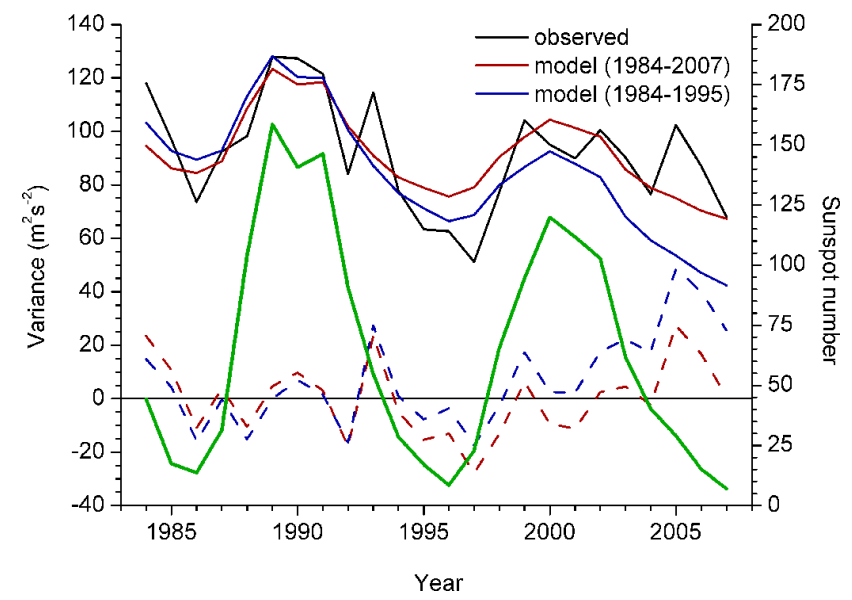

Fig. 1. JJA mean LF GW proxy, and fit including linear trend and a solar cycle according to Eq. (2) at $100 \mathrm{~km}$ virtual height (approx. $91 \mathrm{~km}$ real height). The fit was performed both using the complete dataset 1984-2007 (red curve) and using part of the dataset until 1995 (blue curve). In the lower part the respective residuals are given as dashed lines. The sunspot number is also added as green line.

with $R$ being the 13-monthly smoothed relative sunspot number. We are only interested in long-term and qualitative connections, so employing other widely used solar proxies as F10.7 are not superior to use of $R$ here. As can be seen in the lower part of the figure, the residuals are not normally distributed, and generally the model is not valid during the 1996 as well as the recent solar minimum. Using the same model, but including only data until 1995 (blue line) reveals that the time interval until the early 2000s is well represented by that model, and after 2004 there is a drastic change. We therefore conclude that there is a possible change in dynamical regime since about 2004.

In the case of saturated GWs, linear theory predicts amplitudes proportional to the intrinsic phase speed. Consequently, since GW phase speeds must be positive (eastward) in the summer MLT owing to the filtering effect of the stratospheric and mesospheric easterlies, a negative correlation is expected between the background wind and the GW amplitudes. In Fig. 2 we present GW variance together with the background mean zonal wind at $100 \mathrm{~km}$ virtual height. Note that the background wind is simply the mean of the zonal wind averaged over those times when GW amplitudes have been calculated, and thus may deviate from the prevailing wind. There is an overall anticorrelation between GW variances and zonal winds, as expected from linear theory. However, during solar minimum the correlation reverses. The running correlation (Kodera, 1993) between GW proxy and mean zonal wind is added in the lower part of Fig. 2. Due to the shortness of the time series, only 5 data points are used for each calculation. A clear solar cycle modulation is visible. The running correlation is correlated with the

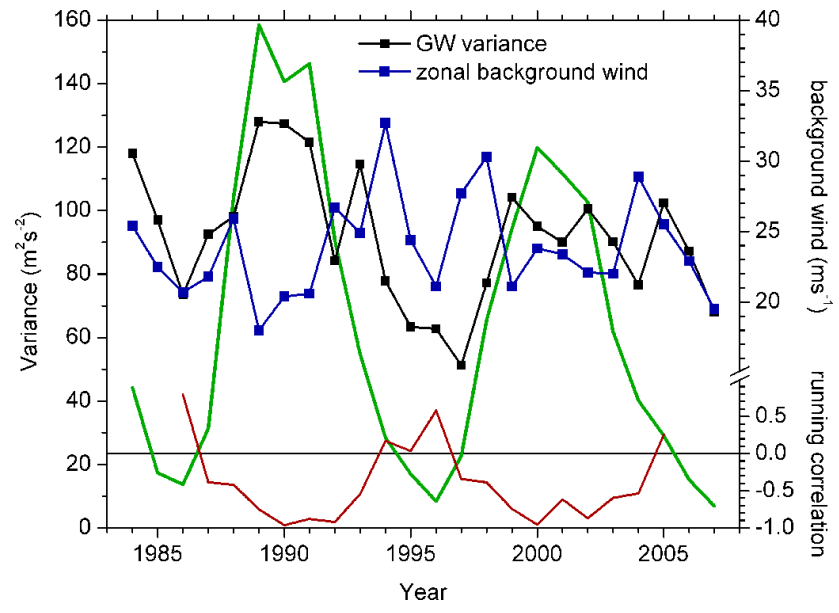

Fig. 2. JJA mean LF GW proxy (black) and zonal mean wind (blue) at $100 \mathrm{~km}$ virtual height. In the lower part of the figure, running correlation coefficients between GW proxy and mean wind are added. The sunspot number is added as green line.

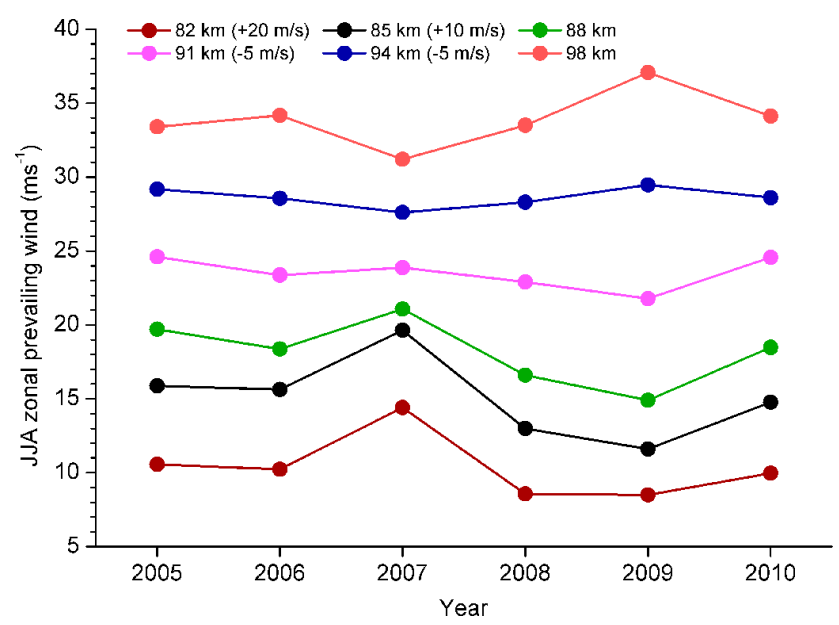

Fig. 3. Collm June-August mean zonal prevailing winds measured by meteor radar.

sunspot number time series with a correlation coefficient of $r=-0.80$. It is also remarkable that this modulation takes place during each solar minimum since 1986 . Note also that the increase of GW variances in 2005, when solar flux already decreases and decreasing $\mathrm{GW}$ variances are expected, has its counterpart in a peak in 1993 . We may conclude that there is obviously a different regime of mean wind-GW coupling during each solar minimum, which is, however, more emphasized during the recent minimum.

LF height measurements at Collm have been terminated in late 2007 , so that the solar minimum is not completely covered by them. To analyze winds and waves during the minimum, in Fig. 3 MR summer mean zonal prevailing winds at 6 height gates are presented. Clearly, interannual variability of winds in the upper and lower height gates is opposite. 


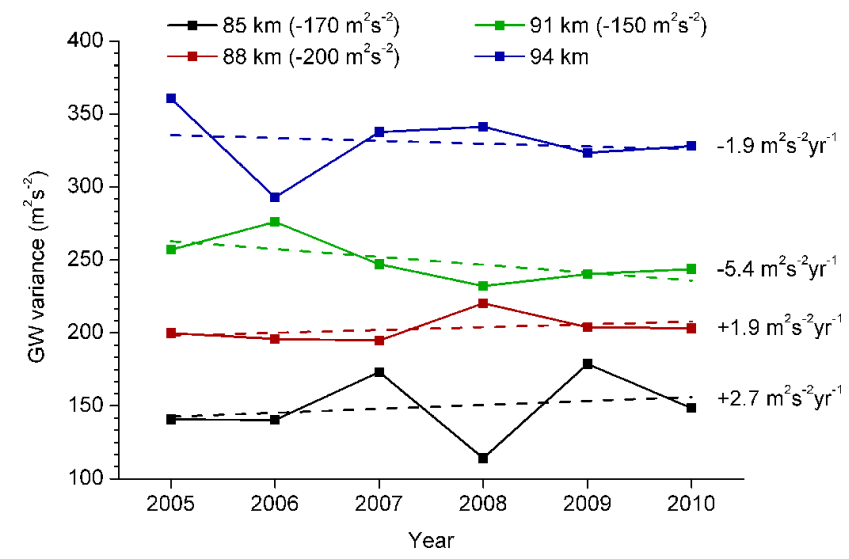

Fig. 4. Horizontal wind variance calculated within 2 -h intervals using Collm MR wind measurements for 4 height gates.

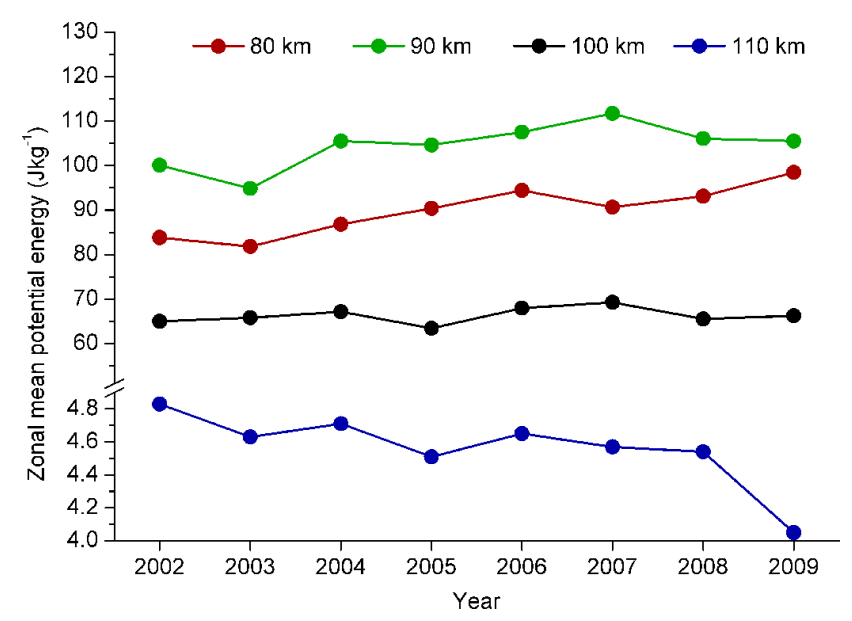

Fig. 5. JJA mean potential energy at $45^{\circ} \mathrm{N}$ from SABER temperature profiles. Data are averages over a $10 \mathrm{~km}$ vertical window, and means over all longitudes.

This is explained by GW acceleration and filtering in the mesosphere. In the case of strong/weak mesospheric easterlies, GW amplitudes are large/small, which then lead to strong/weak vertical wind shear. Figure 3 shows that above $91 \mathrm{~km}$ winds are decreasing until 2007, which is qualitatively consistent with the decrease of the LF winds during 20052007. This also agrees with the zonal LF wind decrease after 1994 (Fig. 2). Note that after 2007, MR winds in the uppermost height gats are increasing again, which would be consistent with the increasing LF winds after 1996 in Fig. 2.

The LF zonal wind decrease during the first part of the solar minimum is accompanied by GW proxy amplitude decrease. MR GW analyses of the recent minimum (Fig. 4) again show this effect qualitatively. The same is the case for SABER potential energy (Fig. 5), although here only the GW amplitudes above $\sim 100 \mathrm{~km}$ decrease with time. This may be due to the fact that we present zonal mean potential

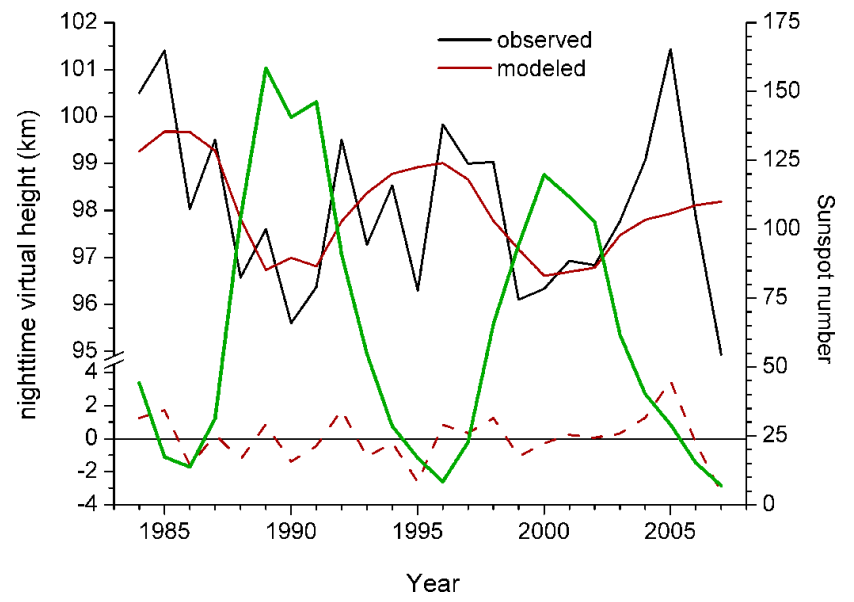

Fig. 6. Collm LF virtual nighttime (22:00-02:00 LT) reflection heights. A linear fit according to Eq. (2) is added as red line, as well as the residuals (red dashed line). The sunspot number is added as green line.

energy, while Figs. 1-4 represent point measurements and non-zonal structures are likely to exist in the MLT. Note that the above mentioned trends are only valid for heights above $90 \mathrm{~km}$, while for the lower height gates the winds and GW amplitudes behave in an opposite manner.

\section{LF virtual heights and mean meteor altitudes}

The solar modulation of correlation between zonal wind and GW amplitudes suggests that the decrease of reference heights during solar minimum of wind systems may play a role. Collm LF virtual nighttime (22:00-02:00 LT) reflection heights are shown in Fig. 6. A multiple linear fit after Eq. (2), but analyzing virtual height instead of variance, is added as red line, as well as the residuals (red dashed line). The sunspot number is added as green line. Note that real heights and height differences are much smaller than virtual height differences. Thus, the strong decrease of LF heights after 2005, for example, represents a real height decrease of about $2 \mathrm{~km}$ only. From the residuals in Fig. 6 one can see that the recent minimum is outstanding. Generally, there is an ionization driven solar cycle of reflection heights such that these are lower during solar maximum than during solar minimum. Thus, the strong decrease after 2005 is unexpected. However, a similar variability has already been observed after 1993 during the last solar minimum, although with much smaller amplitudes, LF reflection height variability is influenced by changes in ionization and mesospheric shrinking. According to Fig. 6 this would mean that during the recent solar minimum thermal shrinking has overcompensated the ionization effect.

Note that the reference height changes shown in Fig. 6 do not show the real variations of a line of constant density, 


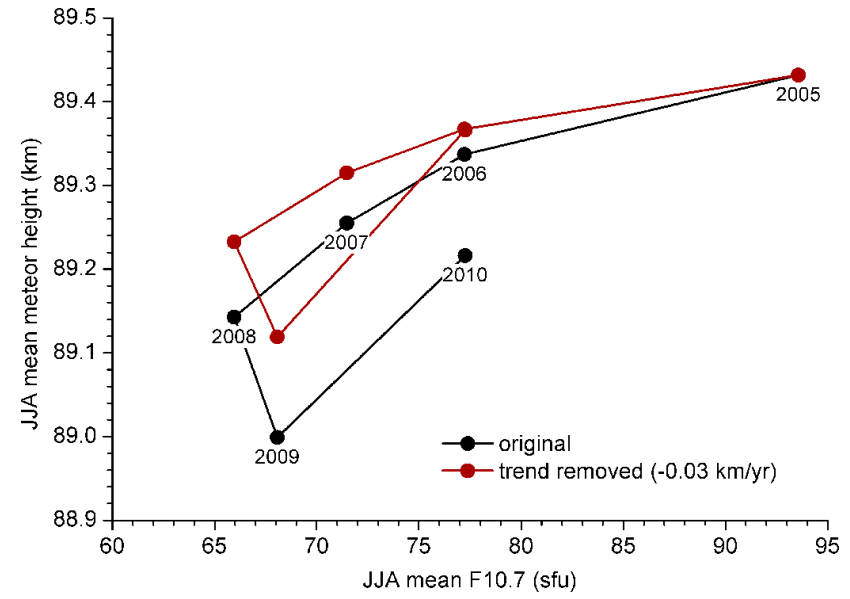

Fig. 7. JJA mean meteor heights over Collm vs. F10.7 solar flux. The red curve represents meteor heights after removing a linear trend of -30 myr $^{-1}$.

for example. A better proxy is the mean height of meteors because these, constant meteor parameters as mass and velocity assumed, burn at a height that is determined by the density distribution. Figure 7 shows June-August mean meteor heights from 2005-2010, plotted against the solar flux. As expected, the meteor heights increase with solar activity. Note, however, that the mean meteor heights during 2008 and 2010, at the same level of solar flux, are different. This is partly due to the fact that the decrease is superposed by the long-term changes of mesospheric density. Bremer and Peters (2008) found a long-term decrease of $-30 \mathrm{myr}^{-1}$ from LF reflection heights (and excluding the solar cycle). Subtracting this from the measured meteor heights in Fig. 7 (red line) shows that then the 2008 and 2010 heights, at the same solar flux, have exactly the same values when long-term cooling of the middle atmosphere is taken into account.

There remains some sort of hysteresis, so that the density increases about one year later than the solar flux does. Similar delay has been found in other parameters, too. A delay of one year of noctilucent cloud occurrence and solar activity has been reported by DeLand et al. (2006) and Bremer et al. (2009). Ortiz de Adler and Elias (2008) showed a similar hysteresis in ionospheric foF2 data. Jacobi et al. (2008) showed that MLT planetary wave activity lags the solar cycle by $1-3$ years.

\section{Discussion and conclusions}

Linear theory predicts that GW amplitudes are proportional to the intrinsic phase speed and thus, in the case of a given GW with specified phase speed, by the zonal wind itself. Therefore a general negative correlation between mean wind and GW amplitudes is expected in the upper mesosphere. Figure 2 indeed shows such a connection for solar maxi- mum. During solar maximum, mesospheric eastward winds are stronger, leading to a negative correlation between solar flux and mean wind there (e.g., Keuer et al., 2007). However, stronger winds and consequently larger GW amplitudes lead to a stronger GW drag, stronger wind shear, and therefore a decreasing solar cycle effect on the mean wind with height, which reverses at some altitude. Such a tendency is visible in the data shown by Keuer et al. (2007) and leads to the reversed interannual variability of the mean winds at lower and upper MR height gates seen in Fig. 3.

The positive correlation between GW amplitudes and zonal winds during solar minimum is unexpected at first glance, but may be explained by a downward shift of the wind systems owing to thermal shrinking of the mesosphere. In such a case, at fixed geometric altitude the reference altitude with respect to the wind system is shifted upward, and therefore the increasing mean wind with decreasing solar flux (which is typical for the mesosphere) changes to a decreasing mean wind with decreasing solar flux, which is typical for the lower thermosphere. This may explain the positive correlation during solar minimum, while there is a negative correlation during the other years, then simply in accordance with linear theory.

The outlined connection for solar minimum cannot be explained by linear theory, at least for the lower thermosphere, because this would, in the case of saturated GWs, always predict negative correlation between GW amplitudes and mean wind at any height. The outlined process also does not explain the change of mean wind tendencies after 2007. Comparison of Figs. 4 and 5 shows that after 2007 decreasing/increasing mean winds are connected with increasing/decreasing GW amplitudes at lower/upper height gates, as expected by linear theory. Thus, there is probably both an effect of GW-mean wind interaction and thermal shrinking, leading to different effects on the mean wind, but for specific years one cannot conclusively predict which one is dominating.

Whether or not the above mentioned coupling processes really work requires more detailed analyses, including more satellite analyses, further radars, and numerical modeling. However, the observations of MLT GWs, mean winds, and reference heights already suggest that there is a height shift during solar minimum which may influence vertical coupling between mesosphere and lower thermosphere. The recent solar minimum represents an extreme case, but the fundamental variability, as shown by the LF measurements, was not qualitatively (although quantitatively) different from the last solar minimum, at least as far as the LF measurements at Collm are concerned. In the thermosphere, however, density decrease during the recent minimum was extreme. Thus, there are still open questions concerning solar variability and its effect on the MLT. 
Acknowledgements. This study was supported by Deutsche Forschungsgemeinschaft under JA 836/22-1. Topical Editor Matthias Förster thanks Edward Kazimirovsky and Suvarna Fadnavis for their help in evaluating this paper.

\section{References}

Bremer, J.: Detection of long-term trends in the mesosphere/lower thermosphere from ground-based radio propagation measurements, Adv. Space Res., 35, 1398-1404, 2005.

Bremer, J. and Berger, U.: Mesospheric temperature trends derived from ground-based LF phase-height observations at midlatitudes: comparison with model simulations, J. Atmos. SolarTerr. Phys., 64, 805-816, 2002.

Bremer, J. and Peters, D.: Influence of stratospheric ozone changes on long-term trends in the meso- and lower thermosphere, J. Atmos. Solar-Terr. Phys., 70, 1473-1481, 2008.

Bremer, J., Hoffmann, P., Latteck, R., Singer, W., and Zecha, M.: Long-term changes of (polar) mesosphere summer echoes, J. Atmos. Solar-Terr. Phys., 71, 1571-1576, 2009.

DeLand, M. T., Shettle, E. P., Thomas, G. E., and Olivero, J. J.: A quarter-century of satellite polar mesospheric cloud observations, J. Atmos. Solar-Terr. Phys., 68, 9-29, 2006.

Emmert, J. T., Lean, J. L., and Picone, J. M.: Record-low thermospheric density during the 2008 solar minimum, Geophys. Res. Lett., 37, L12102, doi:10.1029/2010GL043671, 2010.

Entzian, G.: Der Sonnenfleckenzyklus in der Elektronenkonzentration der D-Region, Kleinheubacher Ber., 12, 309-313, 1967.

Fröhlich, K., Schmidt, T., Ern, M., Preusse, P., de la Torre, A., Wickert, J., and Jacobi, Ch.: The global distribution of gravity wave energy in the lower stratosphere derived from GPS data and gravity wave modelling: Attempt and challenges, J. Atmos. Solar-Terr. Phys., 69, 2238-2248, 2007.

Gavrilov, N. M., Jacobi, Ch., and Kürschner, D.: Climatology of ionospheric drift perturbations at Collm, Germany, Adv. Space Res., 27, 1779-1784, 2001a.

Gavrilov, N. M., Jacobi, Ch., and Kürschner, D.: Short-period variations of ionospheric drifts at Collm and their connection with the dynamics of the lower and middle atmosphere, Phys. Chem. Earth, 26, 459-464, 2001b.

Gray, L. J., Beer, J., Geller, M., Haigh, J. D., Lockwood, M., Matthes, K., Cubasch, U., Fleitmann, D., Harrison, G., Hood, L., Luterbacher, J., Meehl, G. A., Shindell, D., van Geel, B., and White, W.: Solar influences on climate, Rev. Geophys., 48, RG4001, doi:10.1029/2009RG000282, 2010.

Hocking, W. K.: A new approach to momentum flux determinations using SKiYMET meteor radars, Ann. Geophys., 23, 2433-2439, doi:10.5194/angeo-23-2433-2005, 2005.

Jacobi, Ch.: Meteor radar measurements of mean winds and tides over Collm $\left(51.3^{\circ} \mathrm{N}, 13^{\circ} \mathrm{E}\right)$ - comparison with LF drift measurements 2005-2007, Adv. Radio Sci., this issue, 2011.

Jacobi, Ch. and Kürschner, D.: Long-term trends of MLT region winds over Central Europe, Phys. Chem. Earth, 31, 16-21, 2006.

Jacobi, Ch., Gavrilov, N. M., Kürschner, D., and Fröhlich, K.: Gravity wave climatology and trends in the mesosphere/lower thermosphere region deduced from low-frequency drift measurements 1984-2003 (52.1 ${ }^{\circ}$ N, 13.2 ${ }^{\circ}$ E), J. Atmos. Solar-Terr. Phys., 68, 1913-1923, 2006.
Jacobi, Ch., Hoffmann, P., and Kürschner, D.: Trends in MLT region winds and planetary waves, Collm $\left(52^{\circ} \mathrm{N}, 15^{\circ} \mathrm{E}\right)$, Ann. Geophys., 26, 1221-1232, doi:10.5194/angeo-26-1221-2008, 2008.

Keckhut, P., Hauchecorne, A., and Chanin, M. L.: Midlatitude longterm variability of the middle atmosphere: Trends and cyclic and episodic changes, J. Geophys. Res., 100, 18887-18897, 1995.

Keuer, D., Hoffmann, P., Singer, W., and Bremer, J.: Longterm variations of the mesospheric wind field at mid-latitudes, Ann. Geophys., 25, 1779-1790, doi:10.5194/angeo-25-17792007, 2007.

Kodera, K.: Quasi-decadal modulation of the influence of the equatorial quasi-biennial oscillation on the north polar stratospheric temperatures, J. Geophys. Res., 98, 7245-7250, 1993.

Kürschner, D. and Jacobi, Ch.: Quasi-biennial and decadal variability obtained from long-term measurements of nighttime radio wave reflection heights over central Europe, Adv. Space Res. 32, 1701-1706, doi:10.1016/S0273-1177(03)00773-2, 2003.

Kürschner, D., Schminder, R., Singer, W., and Bremer, J.: Ein neues Verfahren zur Realisierung absoluter Reflexionshöhenmessungen an Raumwellen amplitudenmodulierter Rundfunksender bei Schrägeinfall im Langwellenbereich als Hilfsmittel zur Ableitung von Windprofilen in der oberen Mesopausenregion, Z. Meteorol., 37, 322-332, 1987.

Mertens, C. J., Mlynczak, M. G., Lopez-Puertas, M., Wintersteiner, P. P., Picard, R. H., Winick, J. R., Gordley, L. L., and Russell III, J. M.: Retrieval of mesospheric and lower thermospheric kinetic temperature from measurements of $\mathrm{CO}_{2} 15 \mu \mathrm{m}$ earth limb emission under non-LTE conditions, Geophys. Res. Lett., 28, 13911394, 2001.

Mertens, C. J., Schmidlin, F. J., Goldberg, R. A., Remsberg, E. E., Pesnell, W. D., Russell III, J. M., Mlynczak, M. G., LópezPuertas, M., Wintersteiner, P. P., Picard, R. H., Winick, J. R., and Gordley, L. L.: SABER observations of mesospheric temperatures and comparisons with falling sphere measurements taken during the 2002 summer MaCWAVE campaign, Geophys. Res. Lett., 31, L03105, doi:10.1029/2003GL018605, 2004

Ortiz de Adler, N. and Elias, A. G.: Latitudinal variation of $f o \mathrm{~F} 2$ hysteresis of solar cycles 20, 21 and 22 and its application to the analysis of long-term trends, Ann. Geophys., 26, 1269-1273, doi:10.5194/angeo-26-1269-2008, 2008.

Placke, M., Stober, G., and Jacobi, Ch.: Gravity wave momentum fluxes in the MLT - Part I: Seasonal variation at Collm (51.3 ${ }^{\circ} \mathrm{N}, 13.0^{\circ}$ E). J. Atmos. Solar-Terr. Phys., in press, doi:10.1016/j.jastp.2010.07.012, 2011.

Preusse, P., Ern, M., Eckermann, S. D., Warner, C. D., Picard, R. H., Knieling, P., Krebsbach, M., Russell, J. M., Mlynczak, M. G., Mertens, C. J., and Riese, M.: Tropopause to mesopause gravity waves in August: measurement and modelling, J. Atmos. SolarTerr. Phys., 68, 1730-1751, 2006.

Russell III, J. M., Mlynczak, M. G., Gordley, L. L., Tansock, J., and Esplin, R.:: An overview of the SABER experiment and preliminary calibration results, in: Proceedings of the SPIE, 3756, 44th Annual Meeting, Denver, Colorado, 18-23 July, 277-288, 1999.

Solomon, S. C., Woods, T. N., Didkovsky, L. V., Emmert, J. T., and Qian, L.: Anomalously low solar extreme-ultraviolet irradiance and thermospheric density during solar minimum, Geophys. Res. Lett., 37, L16103, doi:10.1029/2010GL044468, 2010. 